\title{
Probing the Multiple Structures of Vaterite through Combined Computational and Experimental Raman Spectroscopy
}

Marco De La Pierre, Raffaella Demichelis, Ursula Wehrmeister, Dorrit E. Jacob, Paolo Raiteri, Julian D Gale, and Roberto Orlando

J. Phys. Chem. C, Just Accepted Manuscript • Publication Date (Web): 28 Oct 2014

Downloaded from http://pubs.acs.org on October 29, 2014

\section{Just Accepted}

"Just Accepted" manuscripts have been peer-reviewed and accepted for publication. They are posted online prior to technical editing, formatting for publication and author proofing. The American Chemical Society provides "Just Accepted" as a free service to the research community to expedite the dissemination of scientific material as soon as possible after acceptance. "Just Accepted" manuscripts appear in full in PDF format accompanied by an HTML abstract. "Just Accepted" manuscripts have been fully peer reviewed, but should not be considered the official version of record. They are accessible to all readers and citable by the Digital Object Identifier (DOI®). "Just Accepted" is an optional service offered to authors. Therefore, the "Just Accepted" Web site may not include all articles that will be published in the journal. After a manuscript is technically edited and formatted, it will be removed from the "Just Accepted" Web site and published as an ASAP article. Note that technical editing may introduce minor changes to the manuscript text and/or graphics which could affect content, and all legal disclaimers and ethical guidelines that apply to the journal pertain. ACS cannot be held responsible for errors or consequences arising from the use of information contained in these "Just Accepted" manuscripts. 


\title{
Probing the Multiple Structures of Vaterite through Combined Computational and Experimental Raman
}

\section{Spectroscopy}

\author{
Marco De La Pierre, ${ }^{1,2}$ Raffaella Demichelis, ${ }^{1, *}$ Ursula Wehrmeister, ${ }^{3}$ Dorrit E. Jacob, ${ }^{4}$ Paolo \\ Raiteri $^{1}$, Julian D. Gale ${ }^{1}$, Roberto Orlando ${ }^{2}$
}

${ }^{1}$ Nanochemistry Research Institute, Department of Chemistry, Curtin University, GPO Box U1987, Perth, WA 6845, Australia

${ }^{2}$ Dipartimento di Chimica, Università degli Studi di Torino and NIS - Nanostructured Interfaces and Surfaces - Centre of Excellence, Via Giuria 7, 10125 Torino, Italy

${ }^{3}$ Institute of Geosciences, Johannes Gutenberg - Universität Mainz, Germany

${ }^{4}$ Department of Earth and Planetary Sciences, Macquarie University, Sydney, NSW, Australia 


\begin{abstract}
First-principles Raman spectra have been computed for several new vaterite structural models that have been recently proposed, and compared with spectra recorded on a set of biogenic, geological and synthetic samples. This set includes new measurements collected on Herdamania momus spicules (Great Barrier Reef, Queensland, Australia), which are known to have purity and crystallinity that are higher than for other biogenic samples. Overall, due to the close structural connection between the various models, the computed Raman spectra are found to be broadly similar. However, the spectra obtained for the two most stable models (monoclinic $C 2$ and trigonal $P 3_{2} 21$, corresponding to two different polytypes of vaterite) exhibit features that are in excellent agreement with the experimental spectra, whereas the other theoretical structures show minor peaks that are not observed experimentally. When comparing the spectra for the two lowest energy structural models $\left(C 2\right.$ and $\left.P 3_{2} 21\right)$, the differences are too small to discriminate between these candidates. The Raman spectrum of Herdamania momus is of higher quality with respect to spectra obtained in previous studies on other biogenic samples. However, there is no significant and systematic difference with respect to samples of geological and synthetic origin.
\end{abstract}

\title{
INTRODUCTION
}

Vaterite is a metastable anhydrous calcium carbonate polymorph often found in nature as a result of biomineralization. ${ }^{1-3}$ In particular, this mineral can appear either as a final polycrystalline product in the hard tissues of living organisms, or most frequently as an intermediate structure during calcium carbonate crystallization. ${ }^{4}$

Before the function of vaterite in the various biomineralization scenarios can be explained, ideally its structural features need to be fully understood. Of all of the numerous models 
proposed during the past 60 years, Meyer's hypotheses (orthorhombic or hexagonal) ${ }^{5,6}$ and Kamhi's hypothesis (hexagonal with partial occupancy of carbonate sites) ${ }^{7}$ have been the most widely accepted and debated for decades, despite the fact that none of their structures can separately explain all spectroscopic and microscopic features that vaterite samples show. ${ }^{8,9}$

In the last five years major advances have been made in understanding the crystallographic structure of vaterite. ${ }^{8,10-14}$ While providing a better, though not definitive, description of vaterite structural features, these studies have also raised a few issues that are still unresolved and may be crucial in understanding the mechanism of some of the various biomineralization pathways. For example, some remaining key questions include the following: How many polytypes of vaterite are there and why? What is their occurrence and why are their crystallites so small? Is vaterite actually chiral? Is it possible to obtain a single crystal, or do all samples contain multiple structures?

In 2009, Wang and Becker ${ }^{10}$ first analyzed and compared the various crystallographic structures that had been proposed for vaterite up to that point, ruling out most of them and proposing a new hexagonal model, with $\mathrm{P}_{5} 22$ symmetry and an expanded unit cell containing $18 \mathrm{CaCO}_{3}$ units. This model was found to be more consistent with spectroscopic data relative to the other prior models, as it allows for independent $\mathrm{CO}_{3}{ }^{2-}$ units within the unit cell. However, Raman spectra recorded on several synthetic, geological and biological samples presented evidence for at least 3 independent carbonate anions to be present in the unit cell, whereas the P6 622 model has only 2. ${ }^{9}$ The earlier orthorhombic and $\mathrm{P} 6_{3} / \mathrm{mmc}$ hexagonal models have also recently been ruled out by Balan et al. ${ }^{15}$ in favor of a new hexagonal model, through simulation of the mixing energy resulting from sulphate substitutions into vaterite and re-analysis of the anomalous data presented by Fernandez-Diaz et al. in $2010 .^{16}$ 
In 2012, two papers presenting seemingly different conclusions were published. In the first of these papers, ${ }^{11}$ by some of the present authors, all of the main ordered structures available in the literature were re-examined by applying electronic structure calculations based on Density Functional Theory (DFT). This work showed that all of them are thermodynamically unstable, representing either a metastable phase (the orthorhombic structures with symmetry $\mathrm{P} 2{ }_{1}{ }_{1} 2_{1}$, resulting from relaxing Meyer's Pbnm model, and Ama2 are higher in energy than the most stable hexagonal* form $\mathrm{P}_{2} 21$ by +2.3 and $+15.1 \mathrm{~kJ} / \mathrm{mol}$ per formula unit, respectively), or a transition state $\left(\mathrm{P}_{5} 22\right)$ between more stable hexagonal or pseudo-hexagonal arrangements $\left(\mathrm{P} 3_{2} 21, \mathrm{P} 6_{5}, \mathrm{P} 112_{1}\right)$. It was also shown that inter-conversion between all of these crystalline phases should be possible at room temperature via rotation of the carbonate anions. In the second publication from 2012, Mugnaioli et al. ${ }^{12}$ showed through automated diffraction tomography that their synthetic samples of vaterite have a monoclinic or triclinic structure belonging to a space group of either $\mathrm{C} 2 / \mathrm{c}$ or $\mathrm{C}-1$. In the same year, Wang and Becker ${ }^{13}$ used molecular dynamics simulation to predict that vaterite could assume either a monoclinic $\mathrm{C} 2 / \mathrm{c}$ structure or a hexagonal $\mathrm{P}_{5} 22$ structure.

A radical change in thinking with regard to the structure of this biomineral has occurred in 2013, as a result of both High-Resolution Transmission Electron Microscopy (HRTEM) on Herdamania momus spicules ${ }^{8}$ and electronic structure calculations. ${ }^{14}$ Kabalah-Amitai et al. ${ }^{8}$ have shown for the first time that two distinct structures are present within the same sample of vaterite, one of which is hexagonal and the other remains of indeterminate symmetry. At the same time, Demichelis et al. ${ }^{14}$ predicted from ab initio calculations that vaterite could exhibit

\footnotetext{
* We classify the structures derived from the P6 622 arrangement as "hexagonal" (though one is trigonal and one is monoclinic) based on the shape of their unit cell rather than on the crystal system.
} 
multiple structures. Here, three almost isoenergetic families of structural configurations were found, with 6-layer hexagonal, 6-layer triclinic $\uparrow$ and 2-layer monoclinic structures. The absolute minimum energy structures for each basin of configurations have $\mathrm{P} 3_{2} 21, \mathrm{C} 1$ (or P1) and C2 symmetry, respectively. Within these basins, which differ in terms of the stacking sequence of the carbonate layers only, carbonate rotations are also energetically possible at room temperature, which leads to an ensemble of structures that may interconvert. This is in agreement with the findings of Kabalah-Amitai et al., ${ }^{8}$ who hypothesized that the arrangement of calcium ions is conserved between their two structures, that the structures are extremely similar, and that they only differ in the relative orientations of the carbonate anions. Some of the possible theoretically predicted structures in the hexagonal basin are intrinsically chiral, and this is also the case for the $\mathrm{C} 1$ and $\mathrm{C} 2$ structures, though this property is hard to demonstrate with currently available experimental techniques, mainly due to the small size of vaterite crystallites.

The only major difference between the findings of Kabalah-Amitai et al. ${ }^{8}$ and Demichelis et $a l .{ }^{14}$ is in regard to the symmetry and unit cell size of vaterite. In particular, the former authors find that their X-ray diffraction (XRD) pattern best fits with Kamhi's original model, ${ }^{7}$ with a small hexagonal unit cell $\left(1 / 9^{\text {th }}\right.$ of the cell employed by Demichelis et al. $\left.{ }^{14}\right)$ where carbonate sites have partial occupancy and an overall symmetry of $\mathrm{P}_{3} / \mathrm{mmc}$. On the contrary, recent studies based on ${ }^{43} \mathrm{Ca}$ NMR spectroscopy and X-ray diffraction show that the only models that can simultaneously explain all the observed features are $\mathrm{C} 2$ and $\mathrm{P} 3_{2} 21 .{ }^{17}$

\footnotetext{
$\dagger$ Despite the conventional choice for this space group being P1, C1 is used because it is obtained from the C-1 arrangement originally proposed by Mugnaioli et al. ${ }^{12}$ This structure is triclinic, however the conventional cell has a quasi-monoclinic shape, with $\alpha \gamma 90^{\circ}$. To avoid further confusion on the structure of vaterite we have decided to keep the notation for the space group unchanged with respect to previous publications. However, we will refer to the $\mathrm{C} 1$ model as triclinic, since the primitive cell that is used for direct comparison with the other models is clearly triclinic.
} 
In this paper we apply Raman spectroscopy, through combining results from computer simulations and experimental measurements, to understand whether this technique is able to provide further insights as to the complex structure of vaterite. We will focus here on the models resulting from Demichelis et al., ${ }^{14}$ leaving the delicate task of re-examining the $\mathrm{P} 6_{3} / \mathrm{mmc}$ model for future investigations. Experimental data presented in Wehrmeister et al. ${ }^{9,18}$ were re-examined in the light of the structural information from Kabalah-Amitai et al. ${ }^{7}$ and Demichelis et al. ${ }^{14}$ and new spectra were recorded. New samples include Herdmania momus spicules from the Great Barrier Reef (Queensland, Australia), the same kind of samples where Kabalah-Amitai et al. ${ }^{8}$ identified two distinct structures.

\section{COMPUTATIONAL METHODS}

All quantum mechanical calculations were performed at the DFT level as implemented in CRYSTAL14. ${ }^{19}$ The PBEsol functional ${ }^{20}$ was used, together with an all-electron Gaussian type basis set already adopted in a number of studies on calcium carbonates, including recent Raman and IR investigations of the polymorphs calcite and aragonite. ${ }^{21,22}$ The accuracy of this approach in describing the structure and properties of vaterite has already been documented in previous works. ${ }^{11,14}$ Geometry optimization and the calculation of vibrational frequencies were performed following the same schemes and adopting the same parameters and thresholds as described in earlier publications. ${ }^{11,14}$

Raman vibrational spectra were computed for all of the seven minimum energy structures listed in Demichelis et al. ${ }^{14}$ Raman intensities were obtained through an analytical approach based on combining gradients of mono- and bi-electronic integrals with a Coupled Perturbed Hartree-Fock/Kohn-Sham (CPHF/KS) scheme for the response of the crystalline orbitals to a 
static dielectric field. Further details on this method can be found in Maschio et al. ${ }^{23,24}$ Isotropic intensities for a polycrystalline sample were derived at the experimental conditions (temperature, laser frequency) according to Prosandeev et $a l .{ }^{25}$ Finally, simulated spectra were generated with inclusion of Lorentzian broadening. Being unable to compute broadening parameters (i.e. Full Width at Half Maximum, FWHM) within the adopted model, a single value of $5 \mathrm{~cm}^{-1}$ was set for all peaks. Although this is not a realistic value for the whole spectral range, it provides a reasonable resolution for the visual inspection of features in the computed spectra.

\section{EXPERIMENTAL METHODS}

All Raman spectra were recorded at room temperature using a HORIBA Jobin Yvon LabRAM HR (high resolution) 800 spectrometer equipped with a Si-based charge-coupled device (CCD) detector (Peltier-cooled), an integrated Olympus BX41 optical microscope and an automatized $x$ $-y$ stage. A $50 \times$ long-distance objective (numerical aperture 0.55 ) and a slit width of $100 \mu \mathrm{m}$ were chosen. The scattered light was dispersed by a grating with 1800 grooves $/ \mathrm{mm}$. Herdmania momus spicules were measured using the $632.816 \mathrm{~nm}$ line of a HeNe laser for excitation with a laser spot size of $c a 2 \times 2 \mu \mathrm{m}$. All spectra were recorded twice. All other spectra were measured using the $532.21 \mathrm{~nm}$ line of a frequency-doubled $\mathrm{Nd}$ :YAG laser and details for these measurements are given in Wehrmeister et al. ${ }^{9,18}$.

The wavenumber accuracy for both setups is $\pm 0.5 \mathrm{~cm}^{-1}$ with a measured spectral resolution of $0.6 \mathrm{~cm}^{-1}$ (FWHM of the Rayleigh line). Spectra were calibrated using the $520.5 \mathrm{~cm}^{-1}$ band of a silicon wafer. Data acquisition and spectra treatment were carried out with the commercially available program LabSpec v4.02 (HORIBA Jobin Yvon GmbH). For all spectra, background subtraction and peak analysis were performed with the software OriginLabPro 7.5 equipped with 
an additional peak-fitting module.

\section{STRUCTURE AND SYMMETRY PROPERTIES}

The structures of the seven models that will be used in this study for vaterite have already been extensively described in two previous publications. We present here a summary mostly focused on their symmetry features.

Vaterite can assume multiple structures belonging to at least three almost isoenergetic basins in the configuration space with different symmetries, namely triclinic, monoclinic and hexagonal (see Table 1). The main difference between these groups of structures is the stacking sequence of the $\mathrm{CO}_{3}{ }^{2-}$ layers. Here the three different orientations of the plane of the carbonate groups within a layer are labeled as A, B and C, with a further' (prime) symbol being used to distinguish between the two possible directions of the $\mathrm{C}-\mathrm{O}$ bonds within this plane. In the hexagonal arrangement, the layers are in an $\mathrm{AC}$ 'BB'CA' sequence, the unit cell has a hexagonal shape and contains 90 atoms. Structures belonging to the monoclinic group have a smaller unit cell (30 atoms) that contains 2 layers of carbonate anions. However, a supercell with hexagonal shape can be built, containing 90 atoms and 6 layers of carbonate units, arranged in an AA'BB'CC' sequence. The structures in the triclinic basin have a quasi-monoclinic conventional cell, with $\alpha \beta 90^{\circ}$. However, for sake of comparison, it is more convenient to refer to the primitive cell, which contains 90 atoms and the sequence of $\mathrm{CO}_{3}{ }^{2-}$ layers is $\mathrm{A}^{\prime} \mathrm{AB} \mathrm{B}^{\prime} \mathrm{CA}$ ' $\mathrm{A}$. The imbalance between the number of each of the six plane types leads to the distortion of the primitive cell away from a hexagonal lattice. Both triclinic structures have $\mathrm{C} 1$ symmetry and they will be referred to in the following sections as $\mathrm{Cl}$ (1) and $\mathrm{Cl}$ (2). A schematic representation of these structures can be found in Demichelis et al. ${ }^{14}$, Figures 1, 2 and Table of Contents. 
Within each basin, interconversion between several structures is possible through rotations of carbonate anions. In Demichelis et al. ${ }^{14}$, we noted that some of the hexagonal structures belong to chiral space groups. In principle, most of the structures in the three basins are chiral, though for low symmetry structures $(\mathrm{C} 1$ and $\mathrm{C} 2)$ both mirror images adopt the same space group.

The main structural features are summarized in Table 1, where the relative stability with respect to the most stable vaterite structure $\left(\mathrm{P} 3_{2} 21\right)$ is also reported, and the relative population of each configuration has been recalculated in order to account for all the possible chiral images. This assumes that a Boltzmann equilibrium distribution exists between configurations, which may not be the case due to kinetic factors. The number of independent $\mathrm{CO}_{3}{ }^{2-}$ units in the unit cell is also reported and ranges from 3 to 18 .

Significantly, previous observations based on Raman spectroscopy have indicated that vaterite has at least 3 independent carbonate units, whereas most of the structures proposed in the literature, apart from the present ones, have no more than $2 .{ }^{9}$ However, the presence of three or more independent carbonate units could also be due to the presence of multiple structures within the same sample, as highlighted by Kabalah-Amitai et al. ${ }^{8}$

\section{THE COMPUTED RAMAN SPECTRA}

The computed Raman spectra of vaterite for all of the hypothetical space groups considered here are reported in Figure 1. Due to the complexity of the vaterite structure, all spectra are very rich, including a large number of peaks with medium to high intensity. Furthermore, because of the close relationship of all of the candidate structures, most of the spectra appear quite similar at first sight. Therefore, it is convenient to divide the various regions as per the work of Wehrmeister et al. ${ }^{9}$ and to analyze each region separately (Table 2). Expanded illustrations of the 
different spectral regions are reported in Figure 2 and Figure 3, and Figures S1 and S2 in the Supplementary Information.

Several theoretical studies have demonstrated that hybrid functionals are a better choice for predicting vibrational properties of minerals ${ }^{26-28}$. However, we have decided to perform this study using the PBEsol functional for sake of consistency with our previous calculations on vaterite, where PBEsol was preferred for providing more realistic thermodynamic predictions. Deviations between computed (PBEsol) and experimental spectra for vaterite have been compared with those obtained for aragonite, which has a well defined structure and for which accurate Raman experiments are available, with peaks assigned and symmetry classified. For all significant regions of the spectrum, we have been able to estimate the systematic shift (reported in Table 2), as well as the error $\left(3 \mathrm{~cm}^{-1}\right)$ based on standard deviation. Further details are available as Supplementary Information). It turns out that, at the PBEsol level, different regions of the Raman spectrum are affected by different systematic deviations from experiment. The computed spectra and frequencies reported here for vaterite have been corrected according to these estimated systematic shifts, and can be considered accurate enough for the purposes of this paper.

As a cross check, Raman spectra have been computed for some of the most stable structures using the hybrid $\mathrm{B}^{2} \mathrm{LYP}^{29}$ approach. As expected, the spectral regions obtained with this functional are not affected by any significant systematic shift ${ }^{21,22,30}$ with respect to the experiment. Overall, the comparison of the B3LYP spectra between the different structures and with the experiments leads to the same qualitative conclusions as obtained with the PBEsol spectra, once the latter have been corrected by the systematic shifts. 
The symmetry classification of the Raman active modes according to the various space groups is reported in Table 3. The region containing the lattice modes, L, shows between 51 and 159 Raman active modes, which appear in the experimental spectra as 8 broad features. The other regions contain a smaller number of Raman active modes: 6 to 18 in the $v_{1}$ and $v_{2}$ regions (with 3 and 1 to 3 peaks usually identified in the experiments, respectively) and 12 to 36 modes in the $v_{3}$ and $v_{4}$ regions ( 2 to 6 experimental peaks for both).

In order to make the computed results comparable with the experimental findings, given the high number of modes in relatively small ranges of wavenumbers, in our analysis peaks have been assumed to be distinct when the difference between their $v_{\max }$ values (see definition below) is at least $5 \mathrm{~cm}^{-1}$. This definition is obviously somewhat arbitrary. However, repeating the classification with different threshold values gives very similar results, and does not change the main spectral features here discussed. Data for frequencies and intensities are reported in Table 4, Table 5 and in Table S3 in the Supplementary Information. Here, each peak is the result of the overlap of $\mathrm{N}_{\mathrm{m}}$ computed modes, and has a frequency $v_{\max }$ corresponding to the frequency of the most intense contributing mode, and an integrated intensity II that is the sum of the $\mathrm{N}_{\mathrm{m}}$ intensities.

\section{Regions $v_{1}$ and $v_{4}$}

The regions containing $\mathrm{CO}_{3}{ }^{2-}$ symmetric stretching $\left(v_{1}\right)$ and in-plane bending $\left(v_{4}\right)$ modes show the most intense and significant peaks, and are reported in Figure 2 and Figure 3 and in Table 4 and Table 5. There are three groups of spectra showing major similarities, especially in the $v_{1}$ region; these arise from the structures with the following symmetries a) $\mathrm{C} 2$ and $\mathrm{P} 3{ }_{2} 21$, b) Cc and $\mathrm{P} 65$, c) $\mathrm{C} 1$ structures and $\mathrm{P} 112_{1}$. Looking at the energetics, we note that structures belonging to these groups of spectra share very similar stabilities. 
In the region of $v_{1}$ all peaks are found in the range $1095-1069 \mathrm{~cm}^{-1}$ and integrated intensities are of the order of hundreds to thousands au (arbitrary units). Table 4 shows that all spectra have features around $1082 \mathrm{~cm}^{-1}$ and $1070 \mathrm{~cm}^{-1}$ (columns 3 and 5 , respectively). The former shows significantly lower intensity for $\mathrm{C} 2, \mathrm{P}_{2} 21$ and $\mathrm{C} 1$ (1) with respect to the other structures, whereas the latter has similar high intensities in all structures.

The configurations C2 and $\mathrm{P} 3_{2} 21$ both lack a peak around $1094 \mathrm{~cm}^{-1}$ (column 1 in Table 4) and a peak around $1079 \mathrm{~cm}^{-1}$ (column 4), with the relative intensity of the other peaks being very similar between the two structures. The $\mathrm{Cc}$ and $\mathrm{P}_{5}$ structures both lack the features around 1087 $\mathrm{cm}^{-1}$ (column 2) and around $1079 \mathrm{~cm}^{-1}$ (column 4), with the remaining peaks having roughly the same intensities. C1 and P112 1 structures have very similar features, apart from C1 (2) lacking the peak around $1079 \mathrm{~cm}^{-1}$ (column 4).

The $v_{4}$ region is reported in Table 5 and in Figure 3. Peaks are found in two distinct subranges, namely $769-740$ and $687-661 \mathrm{~cm}^{-1}$, and intensities are on average much lower than those associated with the $v_{1}$ region, being typically of the order of a few hundred au. In the region 687$661 \mathrm{~cm}^{-1}$ (columns 13-16 in Table 5) the seven structures show very similar features. The main differences are one missing peak in structures $\mathrm{Cc}$ and $\mathrm{P} 6_{5}$ (column 14) and one missing peak for structure $\mathrm{P}_{2} 21$ (column 15). In this region, all structures show their most intense peak around $686 \mathrm{~cm}^{-1}$ (column 13).

The range between 769 and $740 \mathrm{~cm}^{-1}$ (columns 8-12) is more interesting since different structures show different behaviors. The $\mathrm{C} 2$ and $\mathrm{P} 3{ }_{2} 21$ arrangements are the only ones lacking the peak at about $769-765 \mathrm{~cm}^{-1}$ (column 8 ) and having both peaks number 11 and 12; in both cases peak number 9 has an intensity 2-3 times larger than others. The $\mathrm{Cc}$ and $\mathrm{P} 6_{5}$ arrangements exhibit very similar features as in the previous cases, with two missing peaks with respect to the 
other structures. The $\mathrm{C} 1$ and $\mathrm{P} 112_{1}$ structures are the only ones having both peaks number 8 and 9, with the latter being twice as intense as the former. Note also that $\mathrm{P} 3{ }_{2} 21$ is the only structure lacking peak number 10 at around $750 \mathrm{~cm}^{-1}$.

\section{Regions $v_{2}$ and $v_{3}$}

Because of the low intensity, we will comment only briefly on these two regions of the spectrum. The range 900-860 $\mathrm{cm}^{-1}$ (Figure 3 and Table 4) hosts $v_{2}$ out-of-plane bending modes of the carbonate subunits. All structures apart from C2 show only one peak in this region, at about $877-880 \mathrm{~cm}^{-1}$. Structure C2 has two peaks with similar intensity at 881 and $875 \mathrm{~cm}^{-1}$. The range $1540-1360 \mathrm{~cm}^{-1}$ corresponds to $v_{3}$ asymmetric stretching modes of the carbonate anions (Figure $\mathrm{S} 2$ in the Supplementary Information). This region is characterized by a large number of modes having similar low intensities (this spectral feature is noisy or absent in the experiments) and we will not enter into deeper detail in the present discussion.

\section{Lattice modes}

An accurate description of the spectral features of lattice modes would be very challenging for two reasons. Firstly, the spectrum is very complex (up to 160 modes). Secondly, this region is very sensitive to impurities and, at the same time, it falls in a frequency range that is very close to the limit of the edge filter in the experiments, so that comparison with experimental spectra would not be possible. Therefore we will briefly discuss the region of the lattice modes, with the aim of outlining its most relevant features.

Spectra are shown in Figure S1 and the main peaks are listed in Table S3, respectively, in the Supplementary Information. We focus our analysis on two specific sub-ranges where the most intense peaks are found, namely $320-250$ and $130-80 \mathrm{~cm}^{-1}$. To further simplify the analysis, of all the peaks found within these two spectral ranges, Table S3 reports only those with integrated 
intensity larger than 1000 au. The most significant feature of the former region is that all but one structures show a single peak that is far more intense than all others between 311 and $303 \mathrm{~cm}^{-1}$. C2 has two intense peaks with nearly the same intensity, at 305 and $296 \mathrm{~cm}^{-1}$. In the latter region all structures but one show their most intense peak between 115 and $101 \mathrm{~cm}^{-1}$, being instead located at $92 \mathrm{~cm}^{-1}$ in the case of $\mathrm{P} 112_{1}$.

\section{THE EXPERIMENTAL RAMAN SPECTRUM OF HERDMANIA MOMUS}

Herdmania Momus $^{31}$ is a solitary ascidian found as a sessile filter feeder at shallow depths in the temperate and tropical oceans. ${ }^{32}$ It is one of the few known organisms that form vaterite as a normal constituent of their endoskeleton. Each animal contains a large number of vaterite spicules in its body and tunic ranging in size between $100 \mu \mathrm{m}$ and $2.5 \mathrm{~mm} .{ }^{33}$ Most recently, these vaterite spicules were studied in detail by Kabalah-Amitai et al. ${ }^{8}$ Our study obtained new highresolution Raman spectra on spicules from a different individual from the Great Barrier Reef, Australia, allowing for a consistent comparison with previous measurements on other vaterite samples. 9,18

The Raman spectra measured on spicules from this Herdmania Momus sample is shown at the bottom of Figure 1. Expanded spectra for specific ranges are presented at the bottom of Figure 2 and Figure 3 and Figures S2-S3 in the Supplementary Information. Band positions and FWHM values extracted from these measurements are reported in Table 6. In general, the sample does not suffer from the luminescence issues encountered in previous studies on biogenic vaterite. ${ }^{9,18}$ As a result, the spectrum exhibits an improved quality and more bands can be identified. It is worth noticing that the smaller FWHM and the absence of peaks corresponding to calcite and 
aragonite indicate that the sample analyzed in this study could be characterized by higher crystallinity and purity with respect to biogenic samples considered in the past.

Three bands can be identified in the $v_{1}$ region, at 1075,1081 and $1091 \mathrm{~cm}^{-1}$, in line with previous measurements on other biogenic, geologic and synthetic vaterite samples. ${ }^{9,18}$ The $v_{4}$ region shows five bands at $673,686,739,744$ and $751 \mathrm{~cm}^{-1}$, providing a richer picture compared to biogenic samples analyzed in a previous study, ${ }^{9}$ where only 2 to 4 bands could be found. A similar study on other biogenic samples showed the presence of 6 peaks in this region, ${ }^{18}$ as in geologic and synthetic vaterite. ${ }^{9,18}$ The missing band is at $666-668 \mathrm{~cm}^{-1}$ and in the present measurements it could not be distinguished from the one at $673 \mathrm{~cm}^{-1}$. However, note that this region is affected by a large signal-to-noise ratio.

In the lattice mode region, five bands are identified at 176, 209, 268, 302 and $336 \mathrm{~cm}^{-1}$, which lie at similar frequencies compared to previous studies, ${ }^{9,18}$ notably, the corresponding FWHM values are far smaller. The spectral region below $160 \mathrm{~cm}^{-1}$ has not been investigated because it is very close to the edge filter of the instrument. Finally, no peaks can be identified in the $v_{2}$ and $v_{3}$ regions because of the high signal-to-noise ratio. The latter regions exhibited similar problems also in previous studies: one band in region $v_{2}$ was found only in one sample, ${ }^{9}$ and the band in region $v_{3}$ was observed only in Reference ${ }^{18}$ through FT-Raman.

Overall, these measurements on Herdmania Momus exhibit the typical Raman bands found for vaterite samples investigated in the past and coming from different origins (synthetic, geologic, biogenic). Kabalah-Amitai et al. ${ }^{8}$ found that at least two distinct structures were present in Herdmania momus samples, and computer simulation (see Section above) shows that the various possible vaterite structures exhibit very similar Raman features. Therefore, similarities between 
different measurements possibly imply that also other samples analyzed in the past may contain two (or even more) distinct structures.

\section{COMPARISON BETWEEN COMPUTED AND EXPERIMENTAL SPECTRA}

Let us focus on the $v_{1}$ and $v_{4}$ spectral regions, which are the ones where relevant spectral features can be identified. As mentioned in the previous sections, $v_{2}$ has very low intensity in both simulation and experiment; $v_{3}$ has either low intensity (simulation) or noisy broad bands or even no signal at all (experiment); the lattice region is very complex, sensitive to impurities and defects, and close to the limit of the instrument.

In region $v_{1}$ (Table 4 and Table 6 , Figure 2), two computed structures, namely $\mathrm{C} 2$ and $\mathrm{P} 3_{2} 21$, are able to provide a very satisfactory match to the experimental spectrum. They both have 3 peaks, with frequencies in good agreement with the 3 measured bands. Moreover, the distance between extreme peaks $(\Delta)$, i.e. between highest and lowest frequency peaks in the region, is 18 and $16 \mathrm{~cm}^{-1}$, respectively, compared to the experimental value of $16 \mathrm{~cm}^{-1}$. The other five structures all show a peak at around 1095-1093 $\mathrm{cm}^{-1}$ with no corresponding features in the measurements, which results in $\Delta$ values between 22 and $24 \mathrm{~cm}^{-1}$.

Similar observations hold for the $769-740 \mathrm{~cm}^{-1}$ sub-range of $v_{4}$ (Table 5 and Table 6, Figure 3), where computed spectra for $\mathrm{C} 2$ and $\mathrm{P}_{2} 21$ show again great similarity with the experiment. Here the 4 and 3 peaks, respectively, have frequencies in good agreement with the 3 measured bands; $\Delta$ is equal to 16 and $15 \mathrm{~cm}^{-1}$, respectively, to be compared with an experimental value of $12 \mathrm{~cm}^{-1}$. Also in this case, the remaining computed structures show an extra feature at $769-765 \mathrm{~cm}^{-1}$, which raises $\Delta$ to $22-27 \mathrm{~cm}^{-1}$. The second sub-range of this region, $690-650 \mathrm{~cm}^{-1}$, has low intensity in the experiment and nothing that can be used to discriminate between structures in the 
calculations, such that no further information relevant to this discussion can be extracted in this region.

When trying to discriminate between $\mathrm{C} 2$ and $\mathrm{P} 3_{2} 21$, only minor differences are found, which can be summarized by stating that the former shows a larger number of minor peaks than the latter. The most remarkable example is probably the case of the (low intensity) $v_{2}$ region, where $\mathrm{C} 2$ is the only computed structure to show two peaks, separated by $6 \mathrm{~cm}^{-1}$, rather than a single peak. Experimental synthetic samples usually show a larger number of peaks than biogenic ones. However, it is hard to draw conclusions from this observation, as it is also possibly due to factors others than vaterite polytypism, such as the degree of crystallinity and signal-to-noise ratio.

Having computed the free energy differences between the seven structures, we were able to compute the Boltzmann probabilities for each of them (Table 1) and build Boltzmann averaged simulated spectra for the three crystallographic "basins" proposed by Demichelis et al. ${ }^{14}$ However, the three spectra (not reported here) turn out to be very similar, and in all of them the presence of the extra peaks in $v_{1}$ and $v_{4}$ discussed above results in an imperfect agreement with the experimental spectrum.

Overall, the comparison indicates that both the two lowest energy structures proposed in Demichelis et al. ${ }^{14}$, namely $\mathrm{C} 2$ and $\mathrm{P}_{2} 21$, are compatible with Raman measurements on vaterite. This is in strong agreement with a recent study showing that only these two structures are compatible with the observed ${ }^{43} \mathrm{Ca} \mathrm{NMR}$ spectra, and that both structures are compatible with the recorded X-ray diffraction pattern. ${ }^{17}$

Simulated Raman spectra do not make it possible to discriminate as to whether the first, the second or both structures are present in the experimental samples. Nonetheless, C2 and P ${ }_{2} 21$ have hexagonal and monoclinic unit cells, respectively, which is compatible with the recent 
finding by Kabalah-Amitai et $a l^{8}$ of two distinct structures during TEM investigation of Herdmania Momus, one with a hexagonal cell and one still unidentified. The remaining five proposed computer structures seem to be ruled out according to this study. This runs contrary to the room temperature intra-basin structural inter-conversion suggested by Demichelis et al. ${ }^{14}$ on the basis of $\mathrm{CO}_{3}{ }^{2-}$ rotation mechanism, which would result in different domains being present in the same crystal, and thus in peaks from all the structures to appear in the spectra. A possibility is that the system becomes kinetically trapped in a subset of the possible stable structures. To verify this hypothesis, further investigation is required in order to determine the activation barriers for the interconversion between all of the different energy minima.

\section{ASSOCIATED CONTENT}

Supporting Information is available free of charge via the Internet at http://pubs.acs.org."

\section{AUTHOR INFORMATION}

\section{Corresponding Author}

* raffaella.demichelis@curtin.edu.au

\section{Author Contributions}

M.D.L.P., R.D., P.R. and J.D.G. have performed and analyzed the simulations. U.W. and D.E.J. have performed all measures and analyzed the experimental spectra. R.O. has provided a development version of the CRYSTAL code able to perform the calculation of Raman intensities, which M.D.L.P. and R.D. have generalized to include the adopted and other DFT functionals. The manuscript was written through contributions of all authors. All authors have given approval to the final version of the manuscript. 


\section{Notes}

The authors declare no competing financial interest.

\section{ACKNOWLEDGMENTS}

The authors would like to thank the Australian Research Council for Funding through Discovery grant DP0986999 and Future Fellowships FT120100462 and FT130100463, and Curtin University for funding through the Curtin Research Fellowship scheme. iVEC facilities and the Australian National Computing Infrastructure are also acknowledged for the provision of computer time. We are grateful to Carmel McDougall (University of Queensland) for providing us with a suitable sample of Herdmania momus, and to David Quigley (University of Warwick) and Pupa Gilbert (University of Wisconsin-Madison) for fruitful discussions.

\section{SUPPORTING INFORMATION}

This information is available free of charge via the Internet at http://pubs.acs.org.

\section{REFERENCES}

(1) Hasse, B.; Ehrenberg, H.; Marxen, J. C.; Becker, W.; Epple, M. Calcium Carbonate Modifications in the Mineralized Shell of the Freshwater Snail Biomphalaria Glabrata. Chemistry - A European Journal 2000, 6, 3679-3685.

(2) Freeman, C. L.; Harding, J. H.; Quigley, D.; Rodger, P. M. Structural Control of Crystal Nuclei by an Eggshell Protein. Angew Chem Int Ed 2010, 49, 5135-5137. W, G. R.; Sharma, S. K.; Volk, E. Micro-Raman Spectral Study of Vaterite and Aragonite Otoliths of the Coho Salmon,Oncorhynchus kisutchR. Comp. Biochem. Physiol. 1997, 118A, 753-757. Rodriguez-Blanco, J. D.; Shaw, S.; Benning, L. G. The Kinetics and Mechanisms of Amorphous Calcium Carbonate (ACC) Crystallization to Calcite, via Vaterite. Nanoscale 2011, 3, 265-271. Meyer, H. J. Struktur Und Fehlordnung Des Vaterits. Z Kristallogr 1969, 128, 183212.

Meyer, H. J. Uber Vaterit Und Seine Struktur. Angew Chem 1959, 71, 678-679. Kamhi, S. R. On the Structure of Vaterite CaCO3. Acta Cryst 1963, 16, 770-772. Kabalah-Amitai, L.; Mayzel, B.; Kauffmann, Y.; Fitch, A. N.; Bloch, L.; Gilbert, P. U. P. A.; Pokroy, B. Vaterite Crystals Contain Two Interspersed Crystal Structures. Science 2013, 340, 454-457. 
(9) Wehrmeister, U.; Soldati, A. L.; Jacob, D. E.; Häger, T.; Hofmeister, W. Raman Spectroscopy of Synthetic, Geological and Biological Vaterite: a Raman Spectroscopic Study. J. Raman Spectrosc. 2010, 41, 193-201.

(10) Wang, J.; Becker, U. Structure and Carbonate Orientation of Vaterite (CaCO3). Amer Mineral 2009, 94, 380-386.

Demichelis, R.; Raiteri, P.; Gale, J. D.; Dovesi, R. A New Structural Model for Disorder in Vaterite From First-Principles Calculations. CrystEngComm 2012, 14, 44-47.

Mugnaioli, E.; Andrusenko, I.; Schüler, T.; Loges, N.; Dinnebier, R. E.; Panthöfer, M.; Tremel, W.; Kolb, U. Ab Initio Structure Determination of Vaterite by Automated Electron Diffraction. Angew Chem Int Ed 2012, 51, 7041-7045.

Wang, J.; Becker, U. Energetics and Kinetics of Carbonate Orientational Ordering in Vaterite Calcium Carbonate. Amer Mineral 2012, 97, 1427-1436.

Demichelis, R.; Raiteri, P.; Gale, J. D.; Dovesi, R. The Multiple Structures of Vaterite. Crystal Growth \& Design 2013, 13, 2247-2251.

Balan, E.; Blanchard, M.; Pinilla, C.; Lazzeri, M. First-Principles Modeling of Sulfate Incorporation and 34S/32S Isotopic Fractionation in Different Calcium Carbonates. Chemical Geology 2014, 374-375, 84-91.

Fernández-Díaz, L.; Fernández-González, Á.; Prieto, M. The Role of Sulfate Groups in Controlling CaCO3 Polymorphism. Geochimica et Cosmochimica Acta 2010, 74, 6064-6076.

Burgess, K. M. N.; Bryce, D. L. On the Crystal Structure of the Vaterite Polymorph of CaCO3. a Calcium-43 Solid-State NMR and Computational Assessment. Solid State Nuclear Magnetic Resonance 2014, doi: 10.1016/j.ssnmr.2014.08.003.

Wehrmeister, U.; Jacob, D. E.; Soldati, A. L.; Loges, N.; Häger, T.; Hofmeister, W. Amorphous, Nanocrystalline and Crystalline Calcium Carbonates in Biological Materials. J. Raman Spectrosc. 2011, 42, 926-935.

Dovesi, R.; Orlando, R.; Erba, A.; Zicovich-Wilson, C. M.; Civalleri, B.; Casassa, S.; Maschio, L.; Ferrabone, M.; De La Pierre, M.; D’Arco, P.; et al. CRYSTAL14: a Program for the Ab Initio Investigation of Crystalline Solids. Int. J. Quantum Chem. 2014, 114, 1287-1317.

Perdew, J.; Ruzsinszky, A.; Csonka, G.; Vydrov, O.; Scuseria, G.; Constantin, L.; Zhou, X.; Burke, K. Restoring the Density-Gradient Expansion for Exchange in Solids and Surfaces. Phys. Rev. Lett. 2008, 100, 136406.

De La Pierre, M.; Carteret, C.; Maschio, L.; André, E.; Orlando, R.; Dovesi, R. The Raman Spectrum of CaCO3 Polymorphs Calcite and Aragonite: a Combined Experimental and Computational Study. J. Chem. Phys. 2014, 140, 164509. Carteret, C.; De La Pierre, M.; Dossot, M.; Pascale, F.; Erba, A.; Dovesi, R. The Vibrational Spectrum of CaCO3 Aragonite: a Combined Experimental and Quantum-Mechanical Investigation. J. Chem. Phys. 2013, 138, 014201.

Maschio, L.; Kirtman, B.; Rérat, M.; Orlando, R.; Dovesi, R. Ab Initio Analytical Raman Intensities for Periodic Systems Through a Coupled Perturbed HartreeFock/Kohn-Sham Method in an Atomic Orbital Basis. I. Theory. J. Chem. Phys. 2013, 139, 164101.

Maschio, L.; Kirtman, B.; Rérat, M.; Orlando, R.; Dovesi, R. Ab Initio Analytical Raman Intensities for Periodic Systems Through a Coupled Perturbed Hartree- 
Fock/Kohn-Sham Method in an Atomic Orbital Basis. II. Validation and Comparison with Experiments. J. Chem. Phys. 2013, 139, 164102.

(25) Prosandeev, S.; Waghmare, U.; Levin, I.; Maslar, J. First-Order Raman Spectra of AB1/2'B1/2"O3 Double Perovskites. Phys. Rev. B 2005, 71, 214307.

(26) De La Pierre, M.; Orlando, R.; Maschio, L.; Doll, K.; Ugliengo, P.; Dovesi, R. Performance of Six Functionals (LDA, PBE, PBESOL, B3LYP, PBE0, and WC1LYP) in the Simulation of Vibrational and Dielectric Properties of Crystalline Compounds. the Case of Forsterite Mg2SiO4. J. Comput. Chem. 2011, 32, 17751784.

(27) Demichelis, R.; Civalleri, B.; Ferrabone, M.; Dovesi, R. On the Performance of Eleven DFT Functionals in the Description of the Vibrational Properties of Aluminosilicates. Int. J. Quantum Chem. 2010, 110, 406-415.

(28) Valenzano, L.; Torres, F. J.; Doll, K.; Pascale, F.; Zicovich-Wilson, C. M.; Dovesi, R. Ab InitioStudy of the Vibrational Spectrum and Related Properties of Crystalline Compounds; the Case of CaCO 3Calcite. Zeitschrift für Physikalische Chemie 2006, 220, 893-912.

(29) Becke, A. D. Density-Functional Thermochemistry. III. the Role of Exact Exchange. J. Chem. Phys. 1993, 98, 5648.

(30) Valenzano, L.; Torres, F. J.; Doll, K.; Pascale, F.; Zicovich-Wilson, C. M.; Dovesi, R. Ab InitioStudy of the Vibrational Spectrum and Related Properties of Crystalline Compounds; the Case of CaCO 3Calcite. Zeitschrift für Physikalische Chemie 2006, 220, 893-912.

(31) Savigny, M. J. C. Mémoires Sur Les Animaux Sans Vertèbres; Harvard College Library, 1816.

(32) Lowenstam, H. A.; Abbott, D. P. Vaterite: a Mineralization Product of the Hard Tissues of a Marine Organism (Ascidiacea). Science 1975, 188, 363-365.

(33) Lambert, G.; Lambert, C. C. Spicule Formation in the Solitary Ascidian, Herdmania Momus. Journal of Morphology 1987, 192, 145-159. 
Table 1 Classification and symmetry of the seven structures of vaterite considered in the present study.

\begin{tabular}{|c|c|c|c|c|c|c|c|c|c|}
\hline & SGN, HM & $\begin{array}{l}\text { Chiral } \\
\text { image }\end{array}$ & $\begin{array}{l}\text { Crystal } \\
\text { system }\end{array}$ & $\mathrm{N}_{\mathrm{op}}$ & $\mathrm{N}_{\text {at }}$ & $\mathrm{N}_{\mathrm{CO} 3}$ & $\Delta \mathrm{U}_{\mathrm{E}}$ & $\Delta \mathrm{G}$ & $p$ \\
\hline \multirow[t]{2}{*}{ Triclinic } & $1, \mathrm{C} 1(1)$ & $\mathrm{C} 1$ & Triclinic $^{\mathrm{a}}$ & 1 & 90 & 18 & 0.4 & 0.1 & $0.087^{\mathrm{d}}$ \\
\hline & $1, \mathrm{C} 1(2)$ & $\mathrm{C} 1$ & Triclinic $^{\mathrm{a}}$ & 1 & 90 & 18 & 0.5 & 0.2 & $0.083^{\mathrm{d}}$ \\
\hline \multirow[t]{2}{*}{ Monoclinic } & $5, \mathrm{C} 2$ & $\mathrm{C} 2$ & Monoclinic & 2 & 30 & 4 & 0.1 & 0.4 & $0.077^{d}$ \\
\hline & $9, \mathrm{Cc}$ & None & Monoclinic & 2 & 30 & 3 & 0.9 & 1.1 & 0.058 \\
\hline \multirow[t]{3}{*}{ Hexagonal } & $170, \mathrm{P} 6_{5}$ & $\mathrm{P} 6_{1}$ & Hexagonal & 6 & 90 & 3 & 0.7 & 1.0 & $0.060^{\mathrm{d}}$ \\
\hline & $4, \mathrm{P} 112_{1}$ & $\mathrm{P} 112_{1}$ & Monoclinic $^{\mathrm{b}}$ & 2 & 90 & 9 & 0.5 & 0.5 & $0.074^{\mathrm{d}}$ \\
\hline & $153, \mathrm{P}_{2} 21$ & $\mathrm{P} 3{ }_{1} 21$ & Trigonal $^{\mathrm{c}}$ & 6 & 90 & 4 & 0.0 & 0.0 & $0.090^{\mathrm{d}}$ \\
\hline
\end{tabular}

SGN: Space Group Number; HM: Hermann-Mauguin symbol; $\mathrm{N}_{\mathrm{op}}$ : number of symmetry operators; $\mathrm{N}_{\mathrm{at}}$ : number of atoms in the primitive cell; $\mathrm{N}_{\mathrm{CO} 3}$ : number of symmetry-independent $\mathrm{CO}_{3}{ }^{2-}$ units in the unit cell; $\Delta \mathrm{U}_{\mathrm{E}}, \Delta \mathrm{G}$ : electronic energy and free energy differences at $298 \mathrm{~K}$ with respect to $\mathrm{P}_{2} 21\left[\mathrm{~kJ} / \mathrm{mol}\right.$ per formula unit], as calculated in Reference ${ }^{14}$; $p$ : Boltzmann relative populations at $298 \mathrm{~K}$ recalculated to include all mirror images. ${ }^{\mathrm{a}} \alpha \beta 90^{\circ} \mathrm{b}$ a b and $\gamma 120^{\circ}{ }^{\mathrm{c}} \mathrm{a}=\mathrm{b}$ and $\gamma=120^{\circ}$ Population of both mirror images. 
Table 2 Nomenclature for the five regions of the vaterite spectrum and shifts applied to computed frequencies in order to correct for systematic shifts associated with the choice of Kohn-Sham Hamiltonian (estimated based on aragonite data; details available as Supplementary Information).

\begin{tabular}{|l|l|l|l|}
\hline Symbol & Modes & Experimental Range $\left[\mathrm{cm}^{-1}\right]$ & Shift $\left[\mathrm{cm}^{-1}\right]$ \\
\hline $\mathrm{L}$ & Lattice modes & $0-340$ & -3.1 \\
\hline$v_{4}$ & In-plane bending of $\mathrm{CO}_{3}{ }^{2-}$ & $666-685$ & +24.3 \\
& & $738-753$ & \\
\hline$v_{2}$ & Out-of-plane bending of $\mathrm{CO}_{3}{ }^{2-}$ & $873-881$ & +44.4 \\
\hline$v_{1}$ & Symmetric stretching of $\mathrm{CO}_{3}{ }^{2-}$ & $1074-1091$ & +11.4 \\
\hline$v_{3}$ & $\begin{array}{l}\text { Asymmetric stretching of } \\
\mathrm{CO}_{3}{ }^{2-}\end{array}$ & $1421-1555$ & +7.8 \\
\hline
\end{tabular}


Table 3 Symmetry classification of Raman active modes in the seven hypothetical space groups.

\begin{tabular}{|l|c|l|c|l|c|}
\hline & $\mathrm{L}$ & \multicolumn{1}{l|}{$v_{4}$} & $v_{2}$ & $v_{1}$ & \\
\hline $\mathrm{C}^{\mathrm{a}}$ & $159(\mathrm{~A})$ & $36(\mathrm{~A})$ & $18(\mathrm{~A})$ & $18(\mathrm{~A})$ & $36(\mathrm{~A})$ \\
\hline $\mathrm{C} 2$ & $51\left(25 \mathrm{~A}^{\prime}+26 \mathrm{~B}^{\prime \prime}\right)$ & $12\left(6 \mathrm{~A}^{\prime}+6 \mathrm{~B}^{\prime \prime}\right)$ & $6\left(3 \mathrm{~A}^{\prime}+3 \mathrm{~B}^{\prime \prime}\right)$ & $6\left(3 \mathrm{~A}^{\prime}+3 \mathrm{~B}^{\prime \prime}\right)$ & $12\left(6 \mathrm{~A}^{\prime}+6 \mathrm{~B}^{\prime \prime}\right)$ \\
\hline $\mathrm{Cc}$ & $51(24 \mathrm{~A}+27 \mathrm{~B})$ & $12(6 \mathrm{~A}+6 \mathrm{~B})$ & $6(2 \mathrm{~A}+4 \mathrm{~B})$ & $6(4 \mathrm{~A}+2 \mathrm{~B})$ & $12(6 \mathrm{~A}+6 \mathrm{~B})$ \\
\hline $\mathrm{P}_{5}$ & 79 & 18 & 9 & 9 & 18 \\
& $\left(26 \mathrm{E}_{1}+27 \mathrm{E}_{2}+26 \mathrm{~A}\right.$ & $\left(6 \mathrm{E}_{1}+6 \mathrm{E}_{2}+6 \mathrm{~A}\right)$ & $\left(3 \mathrm{E}_{1}+3 \mathrm{E}_{2}+3 \mathrm{~A}\right)$ & $\left(3 \mathrm{E}_{1}+3 \mathrm{E}_{2}+3 \mathrm{~A}\right)$ & $\left(6 \mathrm{E}_{1}+6 \mathrm{E}_{2}+6 \mathrm{~A}\right)$ \\
\hline $\mathrm{P} 112_{1}$ & $159(80 \mathrm{~A}+79 \mathrm{~B})$ & $36(18 \mathrm{~A}+18 \mathrm{~B})$ & $18(9 \mathrm{~A}+9 \mathrm{~B})$ & $18(9 \mathrm{~A}+9 \mathrm{~B})$ & $36(18 \mathrm{~A}+18 \mathrm{~B})$ \\
\hline $\mathrm{P}_{2} 21$ & $78\left(53 \mathrm{E}+25 \mathrm{~A}_{1}\right)$ & $18\left(12 \mathrm{E}+6 \mathrm{~A}_{1}\right)$ & $8\left(6 \mathrm{E}+2 \mathrm{~A}_{1}\right)$ & $10\left(6 \mathrm{E}+4 \mathrm{~A}_{1}\right)$ & $18\left(12 \mathrm{E}+6 \mathrm{~A}_{1}\right)$ \\
\hline
\end{tabular}

Symbols for space groups in Table 1, regions of the spectrum in Table 2. ${ }^{\text {a }}$ Both C1 structures share the same symmetry classification. 
Table 4 Calculated Raman frequencies $\left[\mathrm{cm}^{-1}\right]$ and intensities [au, arbitrary units] of the seven structures considered here for vaterite, in the 1160-1020 and 900-860 $\mathrm{cm}^{-1}$ range $\left(v_{1}\right.$ and $v_{2}$ modes).

\begin{tabular}{|c|c|c|c|c|c|c|c|c|}
\hline & \multicolumn{5}{|c|}{$v_{1}$} & \multicolumn{2}{|c|}{$v_{2}$} \\
\hline & & 1 & 2 & 3 & 4 & 5 & 6 & 7 \\
\hline \multirow[t]{2}{*}{ C1 (1) } & $v_{\max }$ & 1093 & 1086 & $1083 *$ & 1078 & 1071 & 878 & \\
\hline & $\mathrm{II}\left(\mathrm{N}_{\mathrm{m}}\right)$ & $1701(2)$ & $4651(7)$ & $741(4)$ & $438(1)$ & $2074(4)$ & $138(18)$ & \\
\hline \multirow[t]{2}{*}{ C1 (2) } & $v_{\max }$ & 1094 & 1087 & 1083 & & 1070 & 878 & \\
\hline & $\mathrm{II}\left(\mathrm{N}_{\mathrm{m}}\right)$ & $1614(2)$ & $3842(6)$ & $2038(6)$ & & $2112(4)$ & 99 (18) & \\
\hline \multirow[t]{2}{*}{$\mathrm{C} 2$} & $v_{\max }$ & & 1087 & $1083 *$ & & 1069 & 881 & 875 \\
\hline & II $\left(\mathrm{N}_{\mathrm{m}}\right)$ & & $7601(3)$ & $345(2)$ & & 1615 (1) & $26(3)$ & $21(3)$ \\
\hline \multirow[t]{2}{*}{$\mathrm{Cc}$} & $v_{\max }$ & 1094 & & 1081 & & 1072 & 878 & \\
\hline & $\mathrm{II}\left(\mathrm{N}_{\mathrm{m}}\right)$ & $3780(2)$ & & 2984 (2) & & $2838(2)$ & $221(6)$ & \\
\hline \multirow[t]{2}{*}{$\mathrm{P} 6_{5}$} & $v_{\max }$ & 1093 & & 1082 & & 1070 & 877 & \\
\hline & II $\left(\mathrm{N}_{\mathrm{m}}\right)$ & $3836(3)$ & & $2686(3)$ & & 3096 (3) & $88(9)$ & \\
\hline \multirow[t]{2}{*}{$\mathrm{P} 112_{1}$} & $v_{\max }$ & 1095 & 1087 & 1083 & 1079 & 1072 & 878 & \\
\hline & II $\left(\mathrm{N}_{\mathrm{m}}\right)$ & $1357(2)$ & $3747(6)$ & $1430(4)$ & 891 (2) & $2167(4)$ & $142(18)$ & \\
\hline \multirow[t]{2}{*}{$\mathrm{P}_{2} 21$} & $v_{\max }$ & & 1086 & $1082 *$ & & 1070 & 880 & \\
\hline & II $\left(\mathrm{N}_{\mathrm{m}}\right)$ & & 7108 (6) & 799 (2) & & $1672(2)$ & $40(8)$ & \\
\hline
\end{tabular}

For each peak, $v_{\max }$ is the frequency of the most intense contributing mode, II is the integrated intensity, i.e. the sum of the intensities of all the contributing modes, $\mathrm{N}_{\mathrm{m}}$ is the number of contributing modes. Vertical alignment of the peaks is done so to simplify comparison among structures. Peaks marked with an asterisk are not distinct in the spectra, but yet have been reported, as their identification is particularly meaningful for the discussion. 
Table 5 Calculated Raman frequencies and intensities of the seven structures considered here for vaterite, in the $780-640 \mathrm{~cm}^{-1}$ range $\left(v_{4}\right.$ modes $)$.

\begin{tabular}{|c|c|c|c|c|c|c|c|c|c|c|}
\hline & \multicolumn{9}{|c|}{$v_{4}$} \\
\hline & & 8 & 9 & 10 & 11 & 12 & 13 & 14 & 15 & 16 \\
\hline \multirow[t]{3}{*}{ C1 (1) } & $v_{\max }$ & 768 & 756 & 750 & 744 & & 686 & 680 & 671 & 665 \\
\hline & & 179 & 434 & 333 & 469 & & 355 & 66 & 45 & 166 \\
\hline & $\left(\mathrm{N}_{\mathrm{m}}\right)$ & (2) & $(6)$ & (4) & (6) & & (4) & (3) & (3) & (8) \\
\hline \multirow[t]{3}{*}{ C1 (2) } & $v_{\max }$ & 766 & 758 & 751 & 744 & & 685 & $680^{*}$ & 674 & 665 \\
\hline & & 189 & 410 & 186 & 627 & & 357 & 35 & 54 & 178 \\
\hline & $\left(\mathrm{N}_{\mathrm{m}}\right)$ & (2) & $(5)$ & (3) & (8) & & (4) & (2) & (3) & (9) \\
\hline \multirow[t]{3}{*}{$\mathrm{C} 2$} & $v_{\max }$ & & 756 & 751 & 744 & 740 & 686 & 676 & 668 & 661 \\
\hline & & & 578 & 214 & 245 & 367 & 400 & 70 & 123 & 51 \\
\hline & $\left(\mathrm{N}_{\mathrm{m}}\right)$ & & (2) & (1) & (2) & (1) & (2) & (1) & $(2)$ & (1) \\
\hline \multirow[t]{3}{*}{$\mathrm{Cc}$} & $v_{\max }$ & 765 & & 751 & & 740 & 684 & & 669 & 665 \\
\hline & II & 558 & & 340 & & 524 & 377 & & 119 & 123 \\
\hline & $\left(\mathrm{N}_{\mathrm{m}}\right)$ & (2) & & (2) & & (2) & (2) & & $(2)$ & (2) \\
\hline \multirow[t]{3}{*}{$\mathrm{P} 6_{5}$} & $v_{\max }$ & 768 & & 750 & & 743 & 685 & & 671 & 664 \\
\hline & & 488 & & 360 & & 590 & 384 & & 110 & 124 \\
\hline & $\left(\mathrm{N}_{\mathrm{m}}\right)$ & (3) & & (3) & & (3) & (3) & & (3) & (3) \\
\hline \multirow[t]{3}{*}{$\mathrm{P} 112_{1}$} & $v_{\max }$ & 769 & 754 & 749 & & 742 & 686 & 679 & 673 & 664 \\
\hline & & 186 & 516 & 377 & & 337 & 333 & 53 & 77 & 160 \\
\hline & $\left(\mathrm{N}_{\mathrm{m}}\right)$ & (2) & $(8)$ & (4) & & (4) & (4) & (2) & (4) & (8) \\
\hline \multirow[t]{3}{*}{$\mathrm{P} 3_{2} 21$} & $v_{\max }$ & & 758 & & $746 *$ & 743 & 687 & 679 & & 666 \\
\hline & II & & 777 & & 236 & 402 & 335 & 127 & & 172 \\
\hline & $\left(\mathrm{N}_{\mathrm{m}}\right)$ & & (4) & & (3) & (1) & (2) & (3) & & (5) \\
\hline
\end{tabular}

For more details see caption to Table 4. 
Table 6 Experimental Raman band positions and FWHM $\left[\mathrm{cm}^{-1}\right]$ of Herdmania Momus sample.

\begin{tabular}{|l|l|l|l|l|}
\hline $\mathrm{L}$ & $v_{4}$ & $v_{2}$ & $v_{1}$ & $v_{3}$ \\
\hline n.a. below 160 & $673(12.2)$ & n.a. & $1075(4.4)$ & n.a. \\
$176(2.5)$ & $686(1.5)$ & & $1081(6.2)$ & \\
$209(15.0)$ & $739(3.7)$ & & $1091(5.0)$ & \\
$268(20.5)$ & $744(4.6)$ & & & \\
$302(18.5)$ & $751(6.8)$ & & & \\
$336(11.1)$ & & & & \\
\hline
\end{tabular}

n.a., not analyzed because of large signal-to-noise ratio. 
Figure 1 Calculated and experimental Raman spectra of vaterite. Computed spectra are for each of the seven structures considered here. Experimental spectrum measured on Herdamania momus spicules from Great Barrier Reef (Australia).

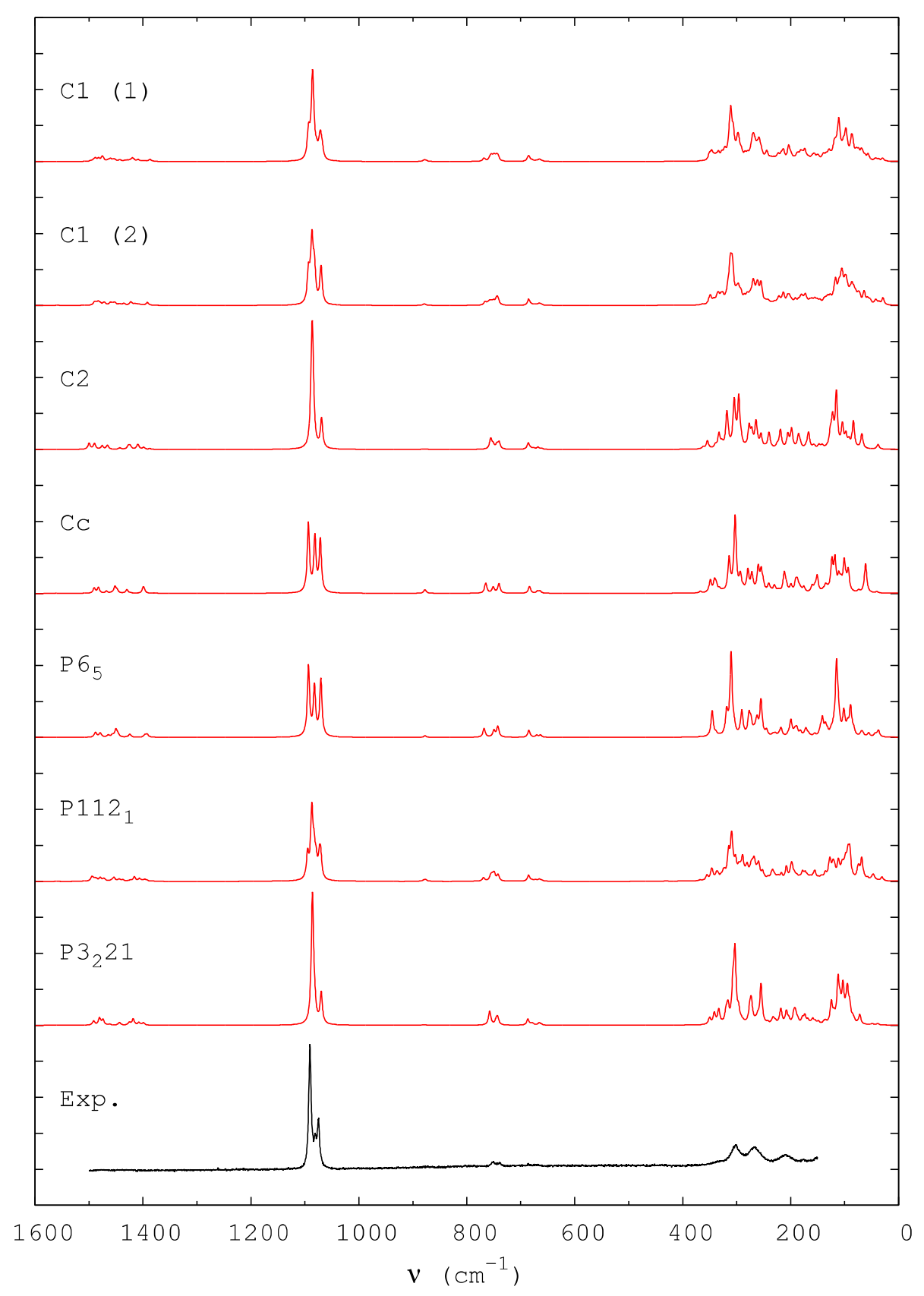

ACS Paragon Plus Environment 
Figure 2 Calculated and experimental Raman spectra of vaterite: expansion of the $1160-1020 \mathrm{~cm}^{-}$ ${ }^{1}$ range $\left(v_{1}\right.$ modes $)$.

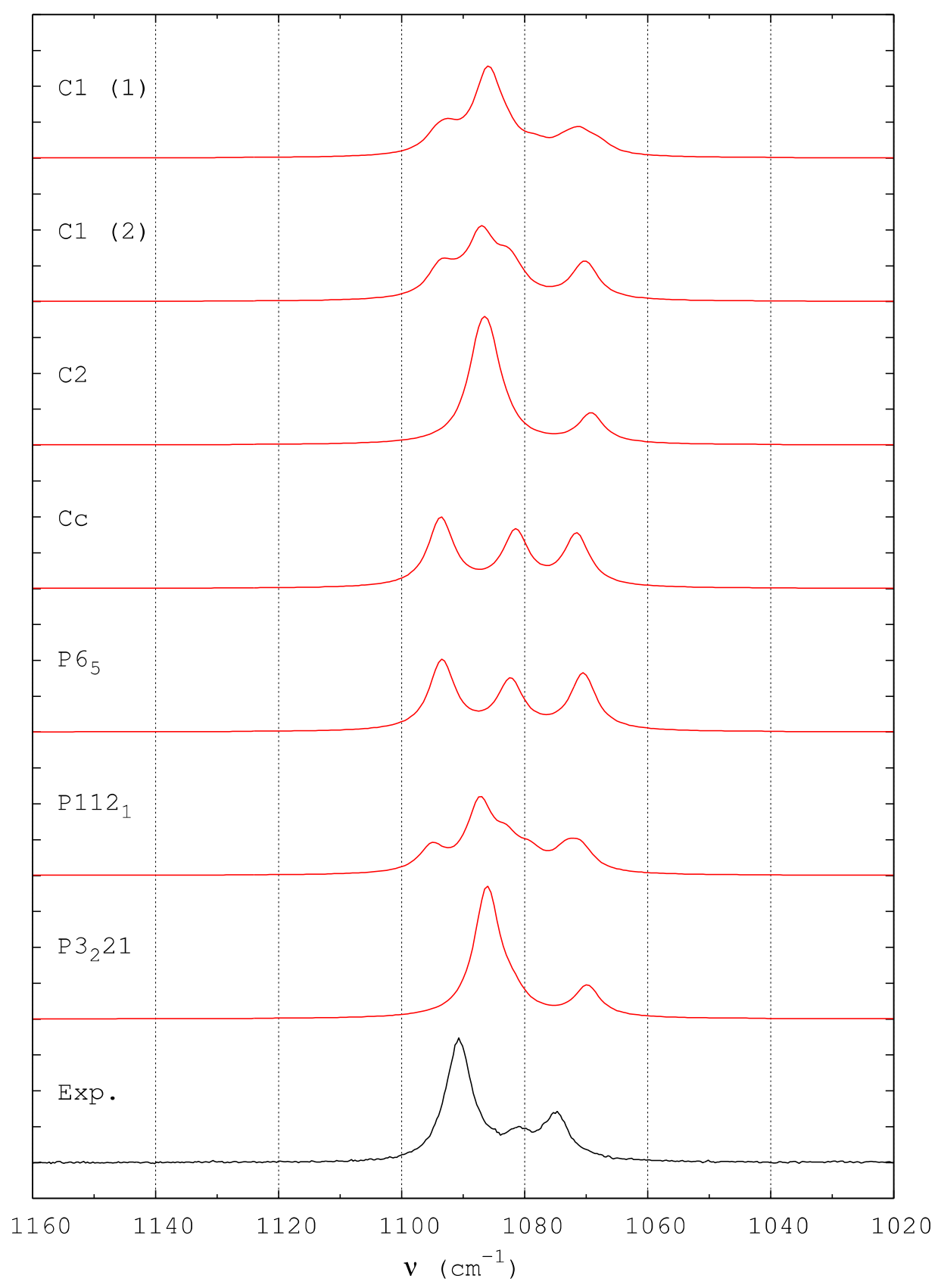


Figure 3 Calculated and experimental Raman spectra of vaterite: expansion of the $900-640 \mathrm{~cm}^{-1}$ range ( $v_{2}$ and $v_{4}$ modes). The experimental spectrum has been magnified by a factor 2 compared to the computed ones.

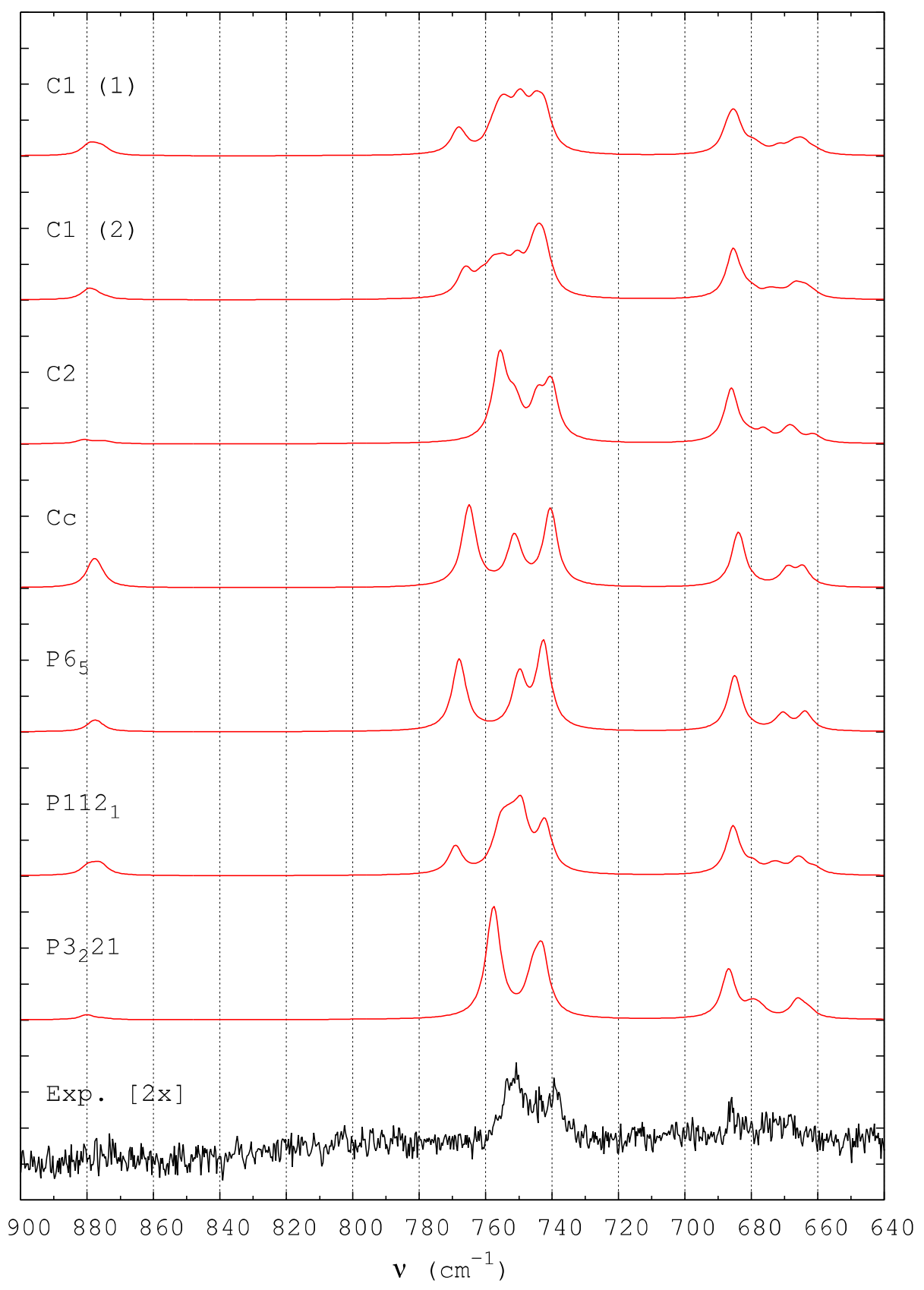

ACS Paragon Plus Environment 
For Table of Contents Only

Despite playing a key role in biomineralization, the structure of vaterite is still debated. We show here that only the two most stable models recently proposed as a result of computer simulation, corresponding to two isoenergetic polytypes of vaterite, exhibit Raman spectra that agree with those measured on a collection of geological, biological and synthetic samples.

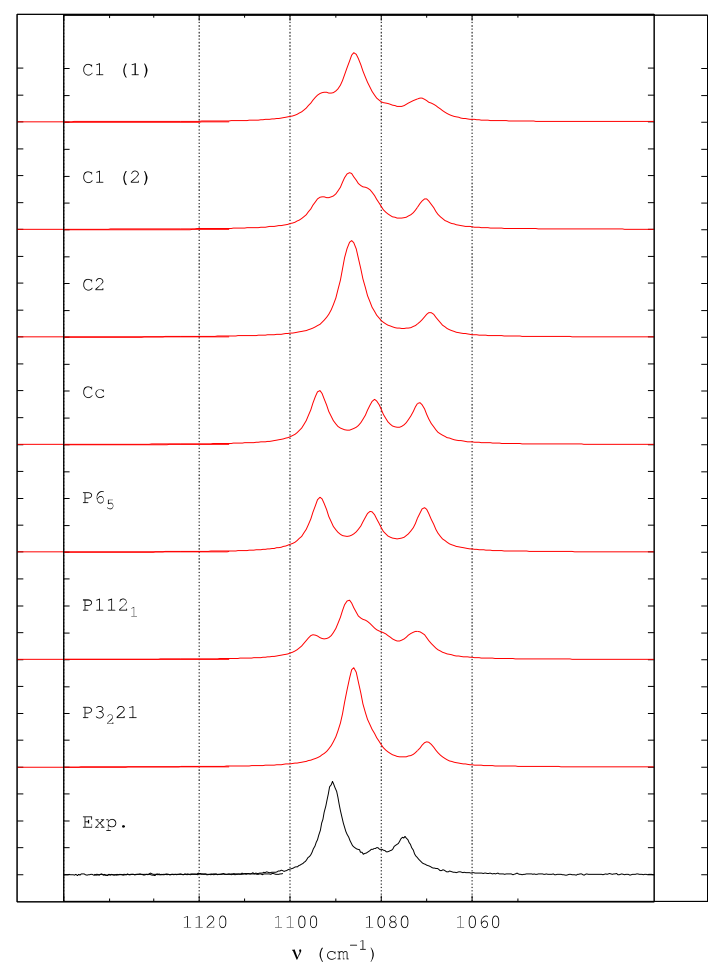

36

37

38

39

40

41

42

43

44

45

46

47

48

49

50

51

52

53

54

55

56

57

58

59

60 